\title{
Improved Transmit Diversity Block Coded OFDM Systems for Highly Dispersive Channels
}

\author{
Bing-Hung Chiang ${ }^{1}$, Ding-Bing Lin ${ }^{2}$, Hsueh-Jyh Li ${ }^{1}$, Jia-Li Wang ${ }^{2}$ \\ 'Graduate Institute of Communication Engineering, National Taiwan University, Taiwan, R.O.C., \\ +886-2-23635251 Ext. 538, hjli@ew.ee.ntu.edu.tw \\ ${ }^{2}$ Institute of Computer, Communication and Control, National Taipei University of Technology, Taiwan, R.O.C., \\ +886-2-2771-2171 Ext. 2274, dblin@ntut.edu.tw
}

\begin{abstract}
In 2000, King proposed two $g_{2}$-based transmit diversity block coded OFDM (TDBC-OFDM) systems, i.e., space-time block coded OFDM (STBC-OFDM) and spacefrequency block coded OFDM (SFBC-OFDM). However, he employed the least square (LS) detector, which was designed under the assumption that the channel is static over the duration of a space-time/frequency codeword. Thereupon, STBC-OFDM/ SFBC-OFDM suffers from highly time/frequency selectivity of the channel. Recently, Antony recommended three novel detectors for space-time block coding (STBC) to combat the rapid channel variation. In our work, these detectors are applied to improve the original $g_{2}$-based TDBC-OFDM systems. Also, the performances of the improved $g_{2}$-based TDBC-OFDM systems are evaluated by computer simulation. Simulation results have revealed that significant performance improvement can be achieved even when the systems are operated in highly dispersive channels.
\end{abstract}

\section{INTRODUCTION}

Space-time block coding (STBC) or transmit diversity block coding (TDBC), an effective transmit diversity technique, was first proposed by Alamouti [1] for flat fading channels. Based on the assumption that the channel is static over the duration of a space-time codeword, it can obtain the same performance as maximum ratio combining (MRC) for receive diversity by least square (LS) detection [2]. Orthogonal frequency division multiplexing (OFDM) is an excellent technique that is capable of reducing the frequency-selective fading into frequencynonselective or flat fading [3]. Thus TDBC can be used for OFDM systems to improve the performance.

According to Alamouti code, $\mathbf{g}_{2}$, King proposed two combinations of TDBC and OFDM, i.e., space-time block coded OFDM (STBC-OFDM) [4] and space-frequency block coded OFDM (SFBC-OFDM) [5]. However, he employed the LS detector, which was designed under the assumption that the channel is static over the duration of a space-time/frequency codeword. Thereupon, STBC-OFDM/SFBC-OFDM suffers from highly time/frequency-selectivity of the channel. Recently, Antony recommended three novel detectors for space-time block coding (STBC) to combat the rapid channel variation. They are zero-forcing (ZF), decision-feedback (DF) and maximum-likelihood (ML) detectors [6].

In this paper, instead of the original LS detector, these novel detectors are applied to improve the performances of $\mathbf{g}_{2}$-based TDBC-OFDM systems. In addition, the performances of the improved $\mathrm{g}_{2}$-based TDBC-OFDM systems are evaluated by computer simulation. Simulation results have revealed that significant performance improvement can be achieved even when the systems are operated in highly dispersive channels. 


\section{TRANSMIT DIVERSITY BLOCK CODED OFDM}

Notice that subsequent description about TDBC-OFDM is based on three assumptions: first, sufficient cyclic prefix (CP) is added to avoid inter-block interference (IBI); second, the channel is constant over one OFDM block duration; third, the channel estimation is perfect. As mentioned before, there are two types of $\mathbf{g}_{2}$-based TDBC-OFDM, namely, STBC-OFDM and SFBC-OFDM. The comparison of these dual systems is shown in Fig. 1.

\section{A. STBC-OFDM}

For STBC-OFDM, two consecutive OFDM blocks, $\mathbf{X}_{2 n+0}$ and $\mathbf{X}_{2 n+1}$, are transmitted from two transmit antennas in two OFDM block durations. Hence, the equivalent STBC transmission matrix for $g_{2}$ is [4]

$$
\mathbf{g}_{2 . S T B C}=\left[\begin{array}{cc}
\mathbf{X}_{2 n+0} & \mathbf{X}_{2 n+1} \\
-\mathbf{X}_{2 n+1}^{*} & \mathbf{X}_{2 n+0}^{*}
\end{array}\right],
$$

where $\quad \mathbf{X}_{2 n+p}=\left[x_{2 n+p, 0} x_{2 n+p, 1} \cdots x_{2 n+p, N_{s}-1}\right]$ for $p=1,2 . \quad x_{2 n+p, k}$ is the transmitted symbol for the $k$ th subcarrier in the $(2 n+p)$ th block, and $N_{s}$ is the number of subcarriers per OFDM block.

In other words, at block instant $(2 n+0), \mathbf{X}_{2 n+0}$ and $\mathbf{X}_{2 n+1}$ are transmitted from antenna 1 and 2, respectively. Then, at block instant $(2 n+1),-\mathbf{X}_{2 n+1}^{*}$ and $\mathbf{X}_{2 n+0}^{*}$ are transmitted from antenna 1 and 2, respectively. Therefore, the system function for STBC-OFDM system can be expressed as

$$
\begin{aligned}
& \mathbf{Y}_{S T B C}=\mathbf{H}_{s T B C} \mathbf{X}_{S T B C}+\mathbf{W}_{S T B C} \\
& {\left[\begin{array}{l}
\mathbf{Y}_{2 n+0} \\
\mathbf{Y}_{2 n+1}^{*}
\end{array}\right]=\left[\begin{array}{ll}
\mathbf{H}_{1,2 n+0} & \mathbf{H}_{2,2 n+0} \\
\mathbf{H}_{2,2 n+1}^{*} & -\mathbf{H}_{1,2 n+1}^{*}
\end{array}\right]\left[\begin{array}{l}
\mathbf{X}_{2 n+0} \\
\mathbf{X}_{2 n+1}
\end{array}\right]+\left[\begin{array}{l}
\mathbf{W}_{2 n+0} \\
\mathbf{W}_{2 n+1}^{*}
\end{array}\right],}
\end{aligned}
$$

where $\quad \mathbf{H}_{i, 2 n+p}=\operatorname{diag}\left[h_{i, 2 n+p, 0} h_{i, 2 n+p, 1} \cdots h_{t, 2 n+p, N_{s}-1}\right]$ for $i=1,2$. $h_{i, 2 n+p, k}$ is the channel frequency response from the $i$ th transmit antenna to the receive antenna for the $k$ th subcarrier in the $(2 n+p)$ th block.

For subcarrier $k$, the detectors consider a pair of symbols, namely, the $n$th space-time codeword as follows.

$$
\begin{aligned}
& \mathbf{y}_{n}=\mathbf{h}_{n} \mathbf{x}_{n}+\mathbf{w}_{n} \\
& {\left[\begin{array}{l}
y_{2 n+0, k} \\
y_{2 n+1, k}^{*}
\end{array}\right]=\left[\begin{array}{ll}
h_{1,2 n+0, k} & h_{2,2 n+0, k} \\
h_{2,2 n+1, k}^{*} & -h_{1,2 n+1, k}^{*}
\end{array}\right]\left[\begin{array}{l}
x_{2 n+0, k} \\
x_{2 n+1, k}
\end{array}\right]+\left[\begin{array}{l}
w_{2 n+0, k} \\
w_{2 n+1, k}^{*}
\end{array}\right] .}
\end{aligned}
$$
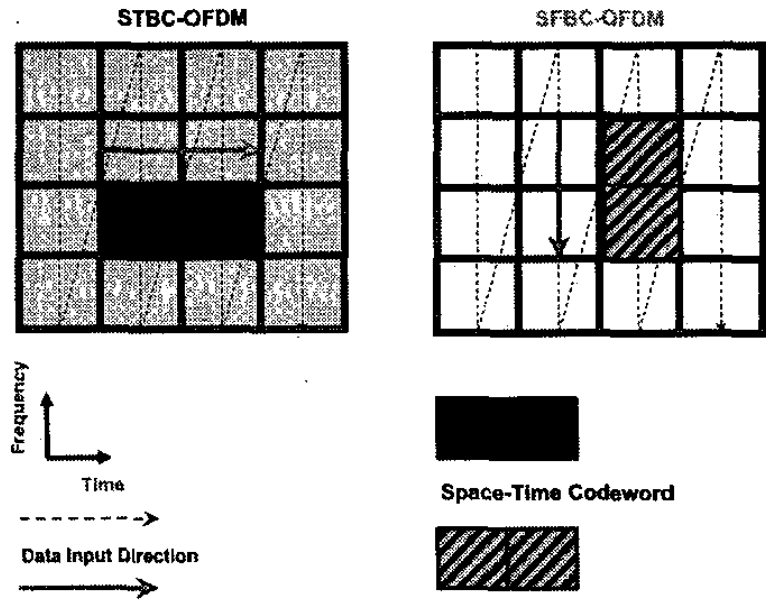

Spatialy tencoding pirtation

Fig. 1 STBC-OFDM VS SFBC-OFDM

1) Least Square Detector: The original LS detector first performs matched filtering on the received symbols, i.e.,

$$
\mathbf{z}_{n}=\mathbf{h}_{n}^{H} \mathbf{y}_{n}=\boldsymbol{\Omega}_{n} \mathbf{x}_{n}+\boldsymbol{\eta}_{n},
$$

where $\mathbf{z}_{n}=\left[\begin{array}{ll}z_{2 n+0, k} & z_{2 n+1, k}\end{array}\right]^{\text {, }}$,

$\boldsymbol{\Omega}_{n}=\mathbf{h}_{n}^{H} \mathbf{h}_{n}=\left[\begin{array}{cc}\left|h_{1.2 n+0, k}\right|^{2}+\left|h_{2,2 n+1, k}\right|^{2} & \beta \\ \beta^{*} & \left|h_{1,2 n+1, k}\right|^{2}+\left|h_{2,2 n+0, k}\right|^{2}\end{array}\right]$,

$\boldsymbol{\eta}_{n}=\mathbf{h}_{n}^{H} \mathbf{w}_{n}$ and $\beta=h_{1,2 n+0, k}^{*} h_{2,2 n+0, k}-h_{1,2 n+1, k}^{*} h_{2,2 n+1, k}$.

It is clear that $\beta$ is the spatial inter-symbol interference (ISI) resulting from rapid channel variation. Nevertheless, the LS detector ignores this spatial ISI and obtains the bad estimates $\hat{x}_{2 n+0, k}$ and $\hat{x}_{2 n+1, k}$ by performing hard-decision on $z_{2 n+0, k}$ and $z_{2 n+1, k}$ independently.

2) Zero-Forcing Detector: The novel $\mathrm{ZF}$ detector forces the spatial ISI to zero without the consideration of noise [6], i.e.,

$$
\dot{\mathbf{z}}_{n}=\mathbf{h}_{n}^{-1} \mathbf{y}_{n}=\mathbf{x}_{n}+\dot{\boldsymbol{\eta}}_{n},
$$

where $\dot{\mathbf{z}}_{n}=\left[\dot{z}_{2 n+0, k} \dot{z}_{2 n+1, k}\right]^{p}$ and $\dot{\eta}_{n}=\mathbf{h}_{n}^{-1} \mathbf{w}_{n}$.

Apparently, the noise component, $\dot{\eta}_{n}$, is correlated, but the $\mathrm{ZF}$ detector disregards the correlation and acquires the suboptimum estimates $\hat{x}_{2 n+0, k}$ and $\hat{x}_{2 n+1, k}$ by performing hard-decision on $\dot{z}_{2 n+0, k}$ and $\dot{z}_{2 n+1, k}$ separately.

3) Decision-Feedback Detector: The novel DF detector benefits from the whitened-matched filtering output [6], i.e., 


$$
\ddot{\mathbf{z}}_{n}=\left(\mathbf{G}_{n}^{H}\right)^{-1} \mathbf{h}_{n}^{H} \mathbf{y}_{n}=\mathbf{G}_{n} \mathbf{x}_{n}+\ddot{\boldsymbol{\eta}}_{n} .
$$

where $\ddot{\mathfrak{\eta}}_{n}=\left(\mathbf{G}_{n}^{H}\right)^{-1} \mathbf{h}_{n}^{H} \mathbf{w}_{n}$,

$$
\mathbf{G}_{n}=\left[\begin{array}{ll}
\frac{\left|h_{1,2 n+0, k} h_{1,2 n+1, k}^{*}+h_{2,2 n+0, k} h_{2,2 n+1, k}^{*}\right|}{\sqrt{\left|h_{1,2 n+1, k}\right|^{2}+\left|h_{2,2 n+0 ., k}\right|^{2}}} & 0 \\
\frac{h_{1,2 n+0, k} h_{2,2 n+0, k}^{*}-h_{1,2 n+1, k} h_{2,2 n+1, k}^{*}}{\sqrt{\left|h_{1,2 n+1, k}\right|^{2}+\left|h_{2,2 n+0 . k}\right|^{2}}} \sqrt{\left|h_{1,2 n+1, k}\right|^{2}+\left|h_{2,2 n+0, k}\right|^{2}}
\end{array}\right]
$$

Notice that $\mathbf{G}_{n}$, a lower triangular matrix with real diagonal elements, is obtained from Cholesky factorization via

$$
\mathbf{h}_{n}^{H} \mathbf{h}_{n}=\mathbf{G}_{n}^{H} \mathbf{G}_{n} .
$$

Accordingly, the estimates of the transmitted symbols can be obtained by means as follows.

$$
\begin{gathered}
\hat{x}_{2 n+0, k}=\operatorname{dec}\left(\ddot{z}_{2 n+0, k}\right), \\
D_{2 n+1, k}=\ddot{z}_{2 n+1, k}-\frac{h_{1,2 n+0, k} h_{2,2 n+0, k}^{*}-h_{1,2 n+1, k} h_{2,2 n+1, k}^{*}}{\sqrt{\left.h_{1,2 n+1, k}\right|^{2}+\left|h_{2,2 n+0, k}\right|^{2}}} \hat{x}_{2 n+0, k} \\
\hat{x}_{2 n+1, k}=\operatorname{dec}\left(D_{2 n+1, k}\right),
\end{gathered}
$$

where $\operatorname{dec}(\cdot)$ denotes hard-decision.

Once the estimate $\hat{x}_{2 n+0, k}$ is not correct, error propagation occurs. Fortunately, it persists for only one space-time codeword.

4) Maximum-Likelihood Detector: The joint ML detector chooses a pair of estimates $\hat{\mathbf{x}}_{n}$ to minimize [6]:

$$
\left\|\mathbf{y}_{n}-\mathbf{h}_{n} \hat{\mathbf{x}}_{n}\right\|^{2} \text {. }
$$

As a result of considering the spatial ISI and noise simultaneously, it obtains the best performance.

\section{B. SFBC-OFDM}

Unlike STBC-OFDM, SFBC-OFDM considers only one OFDM block at a time. Each block is first divided into two parts, namely, $\mathbf{X}_{n, 2 k+0}$ and $\mathbf{X}_{n, 2 k+1}$, and hence the equivalent SFBC transmission matrix for $g_{2}$ is [5]

$$
\mathbf{g}_{2, \mathrm{SFBC}}=\left[\begin{array}{cc}
\mathbf{X}_{n, 2 k+0} & \mathbf{X}_{n, 2 k+1} \\
-\mathbf{X}_{n, 2 k+1}^{*} & \mathbf{X}_{n, 2 k+0}^{\star}
\end{array}\right] \text {, }
$$

where $\mathbf{X}_{n, 2 k+0}=\left[x_{n, 0} x_{n, 2} \cdots x_{n, 2 k+0} \cdots x_{n, N_{s}-2}\right]$,

$$
\mathbf{X}_{n, 2 k+1}=\left[x_{n, 1} x_{n, 3} \cdots x_{n, 2 k+1} \cdots x_{n, N_{s}-1}\right]^{r} \text {, }
$$

for $k=0,1, \ldots,\left(N_{s} / 2-1\right)$.

Therefore, at the first half of each OFDM block duration, $\mathbf{X}_{n, 2 k+0}$ and $\mathbf{X}_{n, 2 k+1}$ are transmitted from antenna 1 and 2, respectively. Then, at the second half of each OFDM block duration, $-\mathbf{X}_{n, 2 k+1}^{*}$ and $\mathbf{X}_{n, 2 k+0}^{*}$ are transmitted from antenna 1 and 2 , respectively. Consequently, the system function for SFBC-OFDM system is expressed as

$$
\begin{aligned}
& \mathbf{Y}_{S F B C}=\mathbf{H}_{S F B C} \mathbf{X}_{S F B C}+\mathbf{W}_{S F B C} \\
& {\left[\begin{array}{c}
\mathbf{Y}_{n, 2 k+0} \\
\mathbf{Y}_{n, 2 k+1}^{*}
\end{array}\right]=\left[\begin{array}{cc}
\mathbf{H}_{1, n, 2 k+0} & \mathbf{H}_{2, n, 2 k+0} \\
\mathbf{H}_{2, n, 2 k+1}^{*}-\mathbf{H}_{1, n, 2 k+1}^{2}
\end{array}\right]\left[\begin{array}{l}
\mathbf{X}_{n, 2 k+0} \\
\mathbf{X}_{n, 2 k+1}
\end{array}\right]+\left[\begin{array}{l}
\mathbf{W}_{n, 2 k+0} \\
\mathbf{W}_{n, 2 k+1}^{2}
\end{array}\right],}
\end{aligned}
$$

where $\mathbf{H}_{i, n, 2 k+0}=\operatorname{diag}\left[h_{i, n, 0} h_{i, n, 2} \cdots h_{i, n, 2 k+0} \cdots h_{i, n, N_{s}-2}\right]$,

$$
\mathbf{H}_{i, n, 2 k+1}=\operatorname{diag}\left[h_{i, n, 1} h_{i, n, 3} \cdot h_{i, n, 2 k+1} \cdot h_{i, n, N_{s}-1}\right],
$$

for $i=1,2$ and $k=0,1, \ldots,\left(N_{s} / 2-1\right)$.

For the $n$th block, the detectors consider a pair of symbols, namely, the $k$ th space-frequency codeword as follows.

$$
\begin{aligned}
& \mathbf{y}_{k}=\mathbf{h}_{k} \mathbf{x}_{k}+\mathbf{w}_{k} \\
& {\left[\begin{array}{l}
y_{n, 2 k+0} \\
y_{n, 2 k+1}^{*}
\end{array}\right]=\left[\begin{array}{cc}
h_{1, n, 2 k+0} & h_{2, n, 2 k+0} \\
h_{2, n, 2 k+1}^{*}-h_{1, n, 2 k+1}^{*}
\end{array}\right]\left[\begin{array}{l}
x_{n, 2 k+0} \\
x_{n, 2 k+1}
\end{array}\right]+\left[\begin{array}{l}
w_{n, 2 k+0} \\
w_{n, 2 k+1}^{*}
\end{array}\right] .}
\end{aligned}
$$

Subsequently, the operations of the detectors for SFBCOFDM are similar to that for STBC-OFDM.

\section{CHANNEL MODEL}

In this paper, the systems are assumed to be operated in the WSSUS Rayleigh fading channel with exponentially decaying power delay profile described as follows [7]. The fading power of the $m$ th path is

$$
\sigma_{m}^{2}=C e^{-m / d} \text { for } m=1,2, . ., M,
$$

where $M$ is the number of paths. Note that $d$ controls the delay spread and $C$ is chosen to satisfy the constraint

$$
\sum_{m=1}^{M} \sigma_{m}^{2}=1
$$

Then solving for $C$ yields

$$
C=\frac{1-e^{-1 / d}}{e^{-1 / d}-e^{-(M+1) / d}} .
$$

It is clear that a small $d$ indicates a small delay spread, and vice versus.

For simplicity, the $T_{s}$-spaced channel model is used here for the simulation of wideband multipath fading channel [7], where $T_{s}$ is the reciprocal of the system bandwidth. Furthermore, each of the uncorrelated taps is of U-shape 
power spectral density and is generated by the mobile fading channel simulator independently [8].

\section{PERFORMANCE RESULTS}

For all simulations, the parameters are as follows. Firstly, the number of uncorrelated paths is 16 and hence the duration of $\mathrm{CP}$ is $16 T_{s}$ seconds; secondly, the number of subcarriers is 64 and hence the total OFDM block duration $80 T_{s}$ seconds; thirdly, the system bandwidth is $20 \mathrm{MHz}$; finally, the modulation is BPSK.

Fig. 2 and Fig. 3 are the simulation results for STBCOFDM and SFBC-OFDM, respectively. As seen from the figures, all detectors obtain similar performances in smoothly dispersive channels, whereas the three novel detectors outperform LS detector significantly in highly dispersive channels. In addition, the error probabilities for ML detectors remain nearly constant with increasing $f_{m} T_{s}$ (normalized Doppler frequency) for STBC-OFDM or with increasing $T_{d, r m s} / T_{s}$ (normalized delay spread) for SFBC-OFDM; indeed, one can conclude that $\mathrm{ML}$ detector is robust to channel selectivity. Furthermore, it is obvious that, with the aid of ML detectors, the error probabilities of TDBC-OFDM systems are much lower than that of conventional OFDM systems.

As shown in Fig. $4 \sim$ Fig. 7, the performances of TDBCOFDM systems are compared in four extreme scenarios, namely, LTLF, HTLF, LTHF and HTHF, respectively. For instant, LTHF stands for low time-dispersion (i.e. frequencyselectivity) and high frequency-dispersion (i.e. timeselectivity). Since STBC-OFDM and SFBC-OFDM are sensitive to frequency-dispersion and time-dispersion, respectively, they obtain similar performance for both LTLF (Fig. 4) and HTHF (Fig. 7). Additionally, STBC-OFDM performs better than SFBC-OFDM for HTLF (Fig. 5), and vice versus (Fig. 6). Again, TDBC-OFDM systems with ML detectors are always of the best performance as a result of considering the spatial ISI and noise simultaneously.

\section{CONCLUSIONS}

In this paper, three novel detectors are applied to TDBC-OFDM systems. To combat the spatial ISI resulting from high channel dispersion, $\mathrm{ZF}$ detector just force the spatial ISI to zero, DF detector alleviates the spatial ISI by whitened-matched filtering and $\mathrm{ML}$ detector reduces the spatial ISI and noise simultaneously. Thereupon, ML detector is of the best performance but highest complexity, while DF and $\mathrm{ZF}$ detectors are of poorer performance but less complexity. However, with the aid of these novel detectors, TDBC-OFDM systems are now compatible with the requirements for wideband wireless applications in highly dispersive channels.

\section{REFERENCES}

[1] S. M. Alamouti, "A simple transmit diversity scheme for wireless communications," IEEE J. Select. Areas Commun., vol. 16, pp. 14151458, Oct. 1998.

[2] D.B. Lin, B.H. Chiang, J.L. Yu, "STBC for wireless communication: a least square interpretation," Proc. of IEEE TENCON '02, Beijing, China, Oct., 2002.

[3] R.van Nee, R. Prasad, OFDM for wireless multimedia communications, Boston: Artech House, 2000

[4] K. F. Lee, D. B. Williams, "A space-time coded transmitter diversity technique for frequency selective fading channels," Proc. IEEE Sensor Array and Multichannel Signal Processing Workshop, pp. 149-152, 2000 .

[5] K. F. Lee, D. B. Williams, "A space-frequency transmitter diversity technique for OFDM systems," Proc. IEEE Global Telecommunications Conference, vol.3, pp. $1473-1477,2000$.

[6] A. Vielmon, Y. (G) Li and J. R. Barry, "Performance of transmit diversity over time-varying Rayleigh-Fading channels," Proc. IEEE Global Telecommunications Conference, pp. 3242-3246, Dec. 2001.

[7] G. L. Stüber, Pricinples of Mobile Communication, $2^{\text {nd }}$ ed. London: Kluwer Academic Publishers, 2001.

[8] M. Pătzold, Mobile Fading Channels. New York: John Wiley \& Sons, 2002 


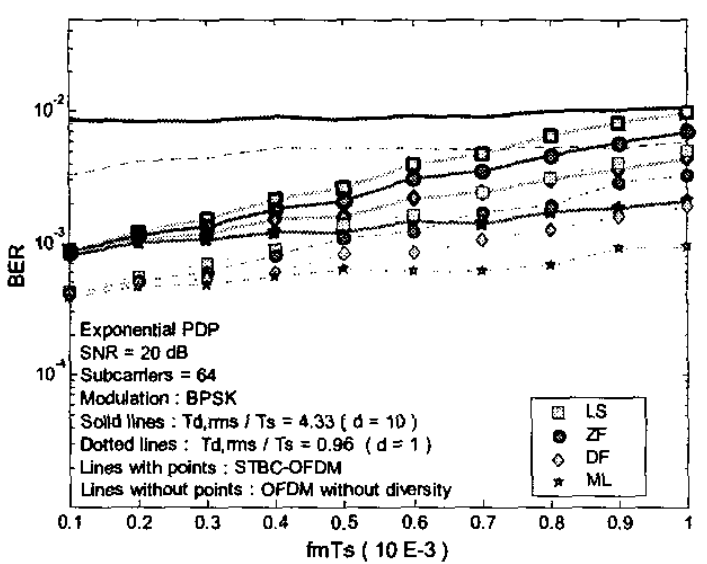

Fig. 2 Performance improvements for STBC-OFDM.

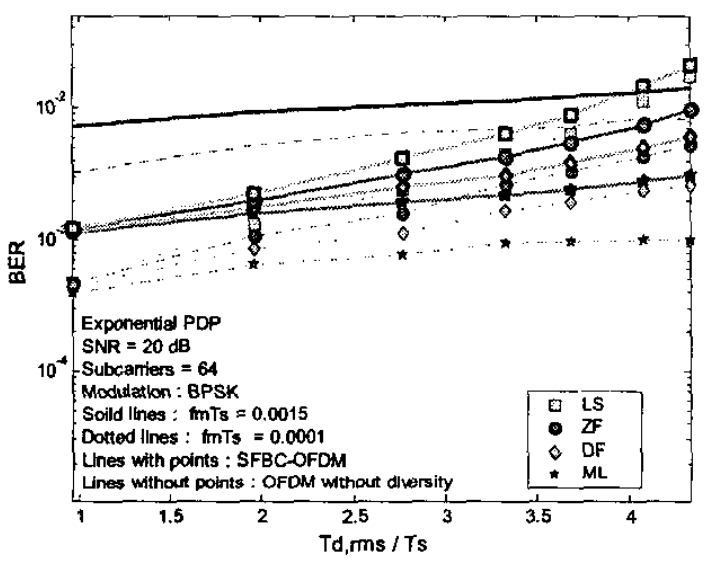

Fig. 3 Performance improvements for SFBC-OFDM.

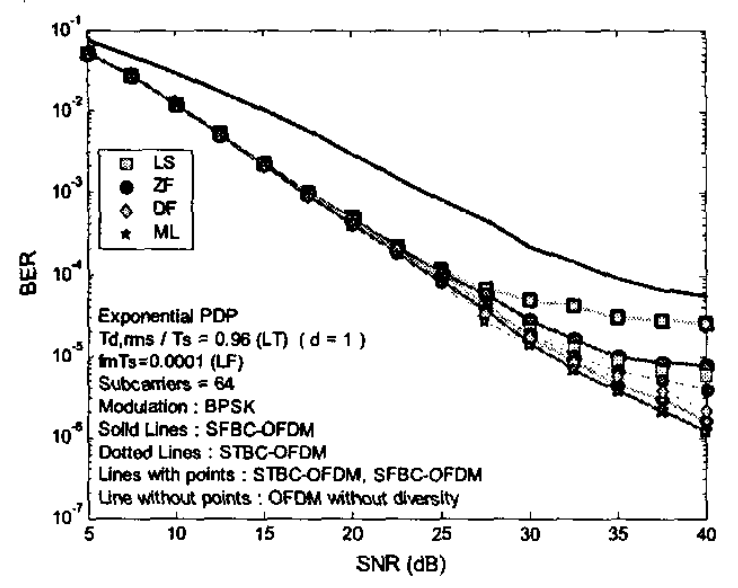

Fig. 4 Performance comparison for LTLF.

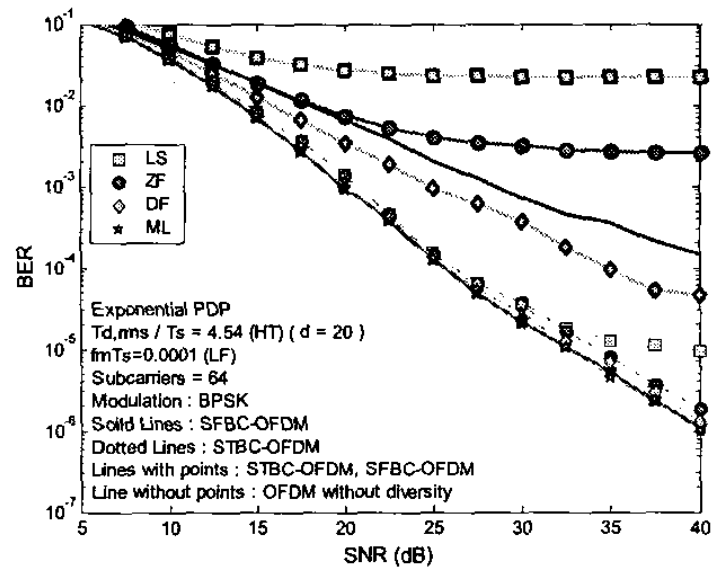

Fig. 5 Performance comparison for HTLF.

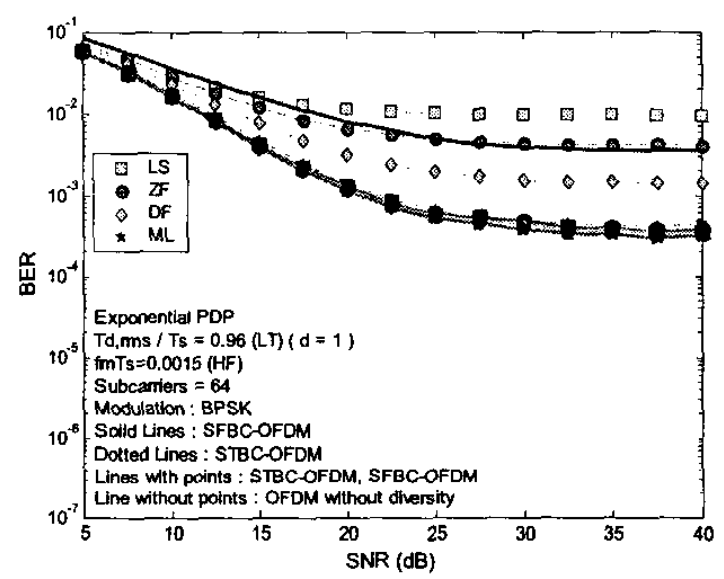

Fig. 6 Performance comparison for LTHF.

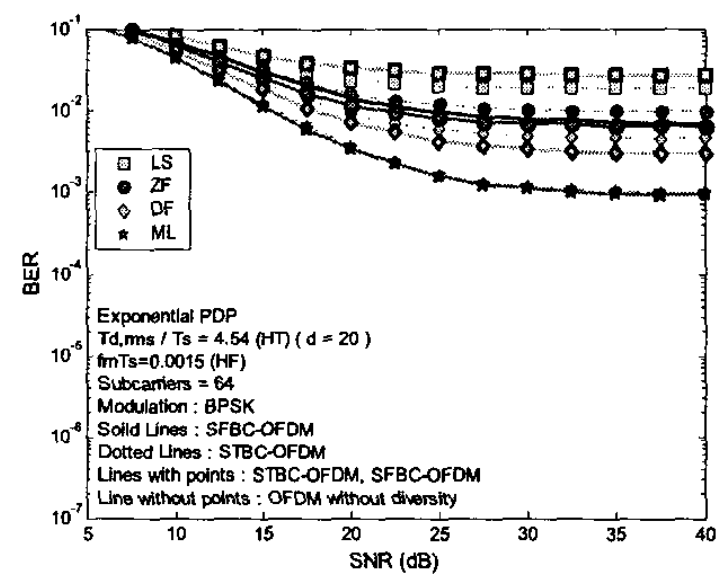

Fig. 7 Performance comparison for HTHF. 